\title{
Post Discharge after Surgery Virtual Care with Remote Automated Monitoring Technology (PVC-RAM): protocol for a randomized controlled trial
}

\author{
Michael H. McGillion RN PhD, Joel Parlow MD MSc, Flavia K. Borges MD PhD, Maura Marcucci MD MSc, \\ Michael Jacka MD MSc, Anthony Adili MD, Manoj M. Lalu MD, Homer Yang MD, Ameen Patel MD, \\ Susan O'Leary MD, Vikas Tandon MD, Gavin M. Hamilton MD MSc, Marko Mrkobrada MD MSc, \\ Carley Ouellette RN MSc, Marissa Bird BSN RN, Sandra Ofori MD, David Conen MD MPH, \\ Pavel S. Roshanov MD MSc, Valerie Harvey BSc, Gordon H. Guyatt MD PhD, Yannick Le Manach MD PhD, \\ Shrikant I. Bangdiwala PhD, Ramiro Arellano MD MSc, Ted Scott PhD, Jennifer Lounsbury RN(EC) MN, \\ Dylan A. Taylor MD, Rahima Nenshi MD MSc, Alan J. Forster MD MSc, Mahesh Nagappa MD, \\ Andre Lamy MD MHSc, Elizabeth Peter PhD RN, Kelsea Levesque BScN RN, Kristen Marosi MD,
} Sultan Chaudhry MD, Shariq Haider MD, Lesly Deuchar MN, Brandi LeBlanc RN, Colin J.L. McCartney MBChB PhD, Emil H. Schemitsch MD, Jessica Vincent MSc, Shirley M. Pettit RN, James Paul MD MSc, Deborah DuMerton RN, Angela Djuric Paulin RN, Marko Simunovic MD MPH, David C. Williams MD MSc, Samantha Halman MD MMed, Christopher M. Schlachta MDCM, Jessica Shelley RN BScN, John Harlock MD, Ralph M. Meyer MD, Michelle Graham MD, Harsha Shanthanna MD PhD, Neil Parry MD, David R. Pichora MD, Haroon Yousef MD MHSc, Husein Moloo MD MSc, Herman Sehmbi MBBS MD, Melissa Waggott RN MScN, Emilie P. Belley-Cote MD PhD, Richard Whitlock MD PhD, P.J. Devereaux MD PhD; on behalf of the PVC-RAM Investigators*

\section{Abstract}

Background: After nonelective (i.e., semiurgent, urgent and emergent) surgeries, patients discharged from hospitals are at risk of readmissions, emergency department visits or death. During the coronavirus disease 2019 (COVID-19) pandemic, we are undertaking the Post Discharge after Surgery Virtual Care with Remote Automated Monitoring Technology (PVC-RAM) trial to determine if virtual care with remote automated monitoring (RAM) compared with standard care will increase the number of days adult patients remain alive at home after being discharged following nonelective surgery.

Methods: We are conducting a randomized controlled trial in which 900 adults who are being discharged after nonelective surgery from 8 Canadian hospitals are randomly assigned to receive virtual care with RAM or standard care. Outcome adjudicators are masked to group allocations. Patients in the experimental group learn how to use the study's tablet computer and RAM technology, which will measure their vital signs. For 30 days, patients take daily biophysical measurements and complete a recovery survey. Patients interact with nurses via the cellular modem-enabled tablet, who escalate care to preassigned and available physicians if RAM measurements exceed predetermined thresholds, patients report symptoms, a medication error is identified or the nurses have concerns they cannot resolve. The primary outcome is number of days alive at home during the 30 days after randomization.

Interpretation: This trial will inform management of patients after discharge following surgery in the COVID-19 pandemic and offer insights for management of patients who undergo nonelective surgery in a nonpandemic setting. Knowledge dissemination will be supported through an online multimedia resource centre, policy briefs, presentations, peer-reviewed journal publications and media engagement. Trial registration: ClinicalTrials.gov, no. NCT04344665

t the start of the coronavirus disease 2019 (COVID-19) pandemic, many hospitals cancelled elective surgeries for various reasons (e.g., to reduce the risk of severe acute respiratory syndrome coronavirus 2 [SARS-CoV-2] transmission, preserve personal protective equipment and maximize bed availability for patients with COVID-19); however, throughout the pandemic, the need for semiurgent (e.g., oncology), urgent (e.g., hip fracture) and emergent
Competing interests: See the end of the article.

*The other members of the PVC-RAM Investigators are listed at the end of the article.

This article has been peer reviewed.

Correspondence to: P.J. Devereaux, p.j.devereaux@phri.ca CMAJ Open 2021. DOI:10.9778/cmajo.20200176 
(e.g., abdominal aortic aneurysm rupture) surgeries has remained. Patients discharged after nonelective (i.e., semiurgent, urgent or emergent) surgeries are at substantial risk of hospital readmissions, presentation to emergency departments or urgent care centres, or death in the 30 days following discharge. ${ }^{1-3}$ Most centres have now resumed elective surgeries. To facilitate management of the backlog of patients waiting for elective surgeries, ensure hospital capacity for patients with COVID-19 and minimize the spread of COVID-19, there is a need to reduce the subsequent use of acute hospital care by patients who undergo nonelective surgery.

A strong rationale and encouraging evidence suggest that virtual care with remote automated monitoring (RAM) will increase the number of days alive at home, in adults discharged after surgery. ${ }^{4}$ We are undertaking the Post Discharge after Surgery Virtual Care with Remote Automated Monitoring Technology (PVC-RAM) randomized controlled trial (RCT) to address the following question: Among adults discharged after nonelective surgery, does virtual care with RAM increase the number of days alive at home during the first 30 days after randomization, compared with standard care?

\section{Methods}

\section{Design and setting}

PVC-RAM is a multicentre, parallel-group, superiority RCT of 900 patients being discharged from the hospital after nonelective surgery. PVC-RAM will determine the effects of virtual care with RAM compared with standard care. Eight Canadian academic tertiary care hospitals are participating (Table 1). Figure 1 reports the trial flow chart.

\section{Trial population}

We are including patients who are 40 years of age or older; have undergone same-day or inpatient nonelective surgery and are being discharged home or are within 24 hours after discharge home and have not had acute hospital care since

\begin{tabular}{|ll|}
\hline $\begin{array}{l}\text { Table 1: Canadian hospitals participating in the PVC-RAM } \\
\text { trial }\end{array}$ \\
\hline Hospital & \multicolumn{1}{c|}{ Location } \\
\hline $\begin{array}{l}\text { Hamilton Health Sciences, Hamilton } \\
\text { General Hospital }\end{array}$ & Hamilton, Ontario \\
\hline $\begin{array}{l}\text { Hamilton Health Sciences, Juravinski } \\
\text { Hospital and Cancer Centre }\end{array}$ & Hamilton, Ontario \\
\hline Kingston Health Sciences & Kingston, Ontario \\
\hline London Health Sciences, Victoria Hospital & London, Ontario \\
\hline $\begin{array}{l}\text { London Health Sciences, University } \\
\text { Hospital }\end{array}$ & London, Ontario \\
\hline \begin{tabular}{l} 
St. Joseph's Healthcare Hamilton \\
\hline The Ottawa Hospital
\end{tabular} & Hamilton, Ontario \\
\hline $\begin{array}{l}\text { University of Alberta Hospital } \\
\text { Note: PVC-RAM = Post Discharge after Surgery Virtual Care with Remote } \\
\text { Automated Monitoring technology. }\end{array}$ & Ontario \\
\hline
\end{tabular}

discharge; and provide informed consent to participate. Box 1 reports the exclusion criteria. Patients with COVID-19 are not excluded from the trial.

\section{Patient recruitment}

Study personnel are using efficient recruitment strategies previously used in perioperative trials. ${ }^{5,6}$ These include identifying eligible patients through screening of daily surgical lists, surgical wards and intensive care units. Investigators ask clinicians working in anesthesiology, surgery and medicine to contact the study personnel regarding patients who have undergone nonelective surgery. Research personnel approach eligible patients after surgery to obtain written informed consent (Appendix 1A, available at www.cmajopen.ca/content /9/1/E142/suppl/DC1). Study personnel may obtain consent via telephone if the patient has already been discharged home and they are within 24 hours of discharge.

\section{Randomization and blinding}

Randomization occurs when a patient is deemed eligible and informed consent is obtained. Patients are only randomly assigned to a study group after the most responsible physician has decided to discharge the patient home. Although our goal is to assign patients randomly before hospital discharge, some patients may be discharged before study personnel can randomly assign the patient.

Research personnel randomly assign patients to a study group via an interactive $W$ eb randomization system. This 24-hour Internet-based randomization system is maintained at the Population Health Research Institute (PHRI), which is part of Hamilton Health Sciences and McMaster University in Hamilton, Ontario, Canada. The randomization procedure ensures assignment concealment from the study personnel and investigators who are recruiting and randomly assigning patients.

The randomization process uses block randomization stratified by centre and type of surgery (i.e., cardiac v. noncardiac). We use randomly varying block sizes, and study personnel and investigators do not know the block sizes. We randomly assign patients in a $1: 1$ fashion to receive virtual care with RAM or standard care. Patients, health care providers and data collectors

\section{Box 1: Exclusion criteria for the PVC-RAM trial}

- Patient underwent same-day surgery and the surgeon or anesthesiologist believe the case was not a typical inpatient surgery managed as a same-day surgery because of the COVID-19 pandemic

- Patient went to rehabilitation or convalescent care and stayed there for more than $7 \mathrm{~d}$ after undergoing surgery

- Patient was unable to communicate with research staff, complete study surveys or undertake an interview using a tablet computer because of a cognitive, language, visual or hearing impairment

- Patient resided in an area without cellular network coverage

Note: Any patients who met at least 1 of these criteria were ineligible to participate in the trial. COVID-19 = coronavirus disease 2019, PVC-RAM = Post Discharge after Surgery Virtual Care with Remote Automated Monitoring Technology. 


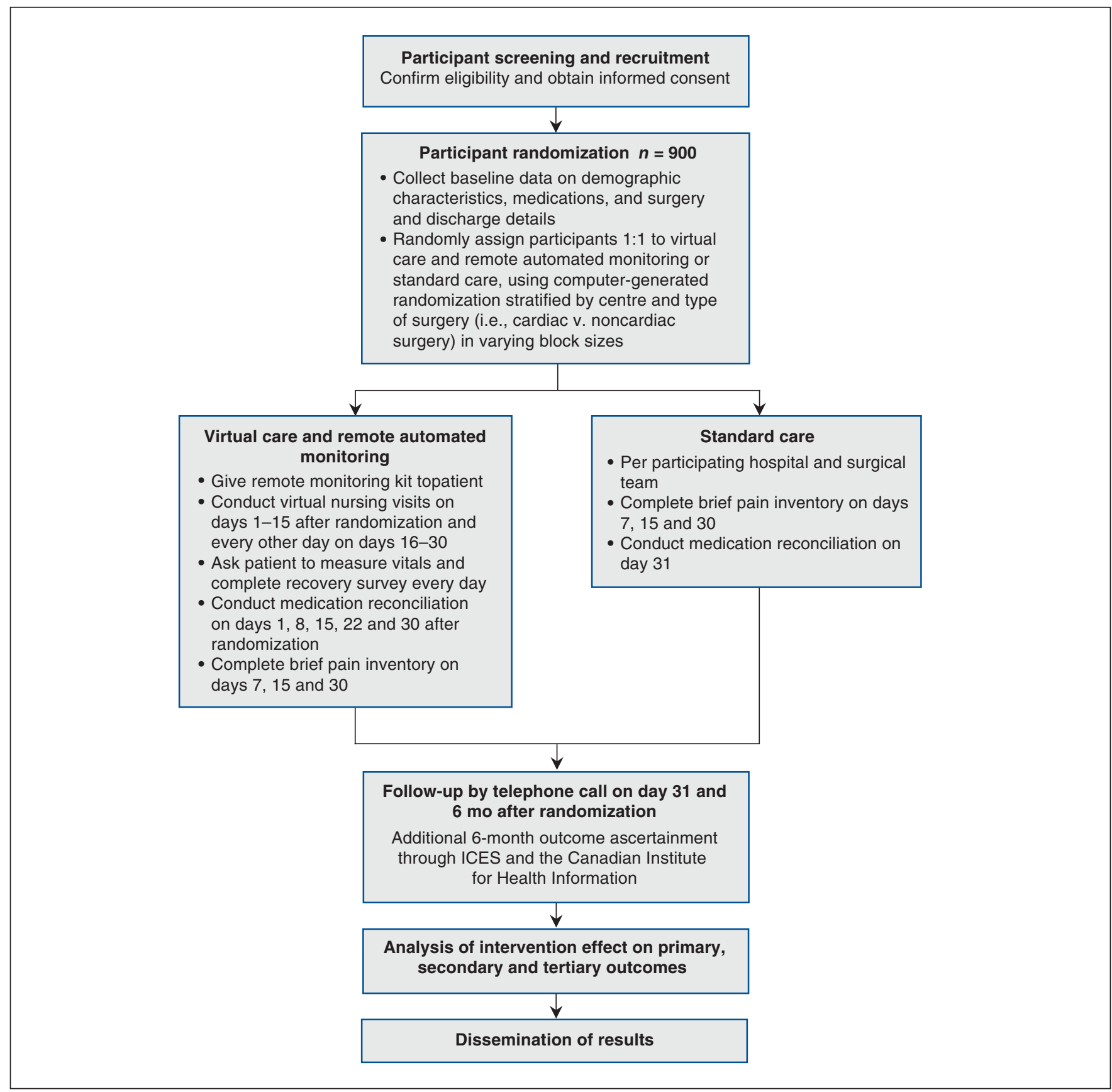

Figure 1: Trial flow chart.

are aware of patients' group assignment. Outcome adjudicators are masked to group assignment.

\section{Trial interventions}

Patients are randomly assigned to receive virtual care with RAM for 30 days after randomization or standard care. The RAM technology used is the Cloud DX Connected Health Kit, consisting of a Bluetooth-enabled, Health Canada-licensed blood pressure cuff, pulse oximeter, temperature probe and weight scale. Each of these devices is paired with a preprogrammed, cellular modem-enabled Samsung tablet computer. Published user testing data show the ease of use of the
Connected Health Kit by patients (including older patients) who do not have prior experience with tablets or computers. ${ }^{7}$ Further details on the components, validation, testing and security features of this technology as well as the training that nurses and physicians received to use it are available in Appendix 1B.

Research staff teach patients assigned to receive virtual care with RAM how to use the cellular modem-enabled tablet computer and RAM technology from Cloud DX (Figure 2). Daily for 30 days, patients take biophysical measurements and complete a recovery survey, and nurses review these results (Appendix 1C). 


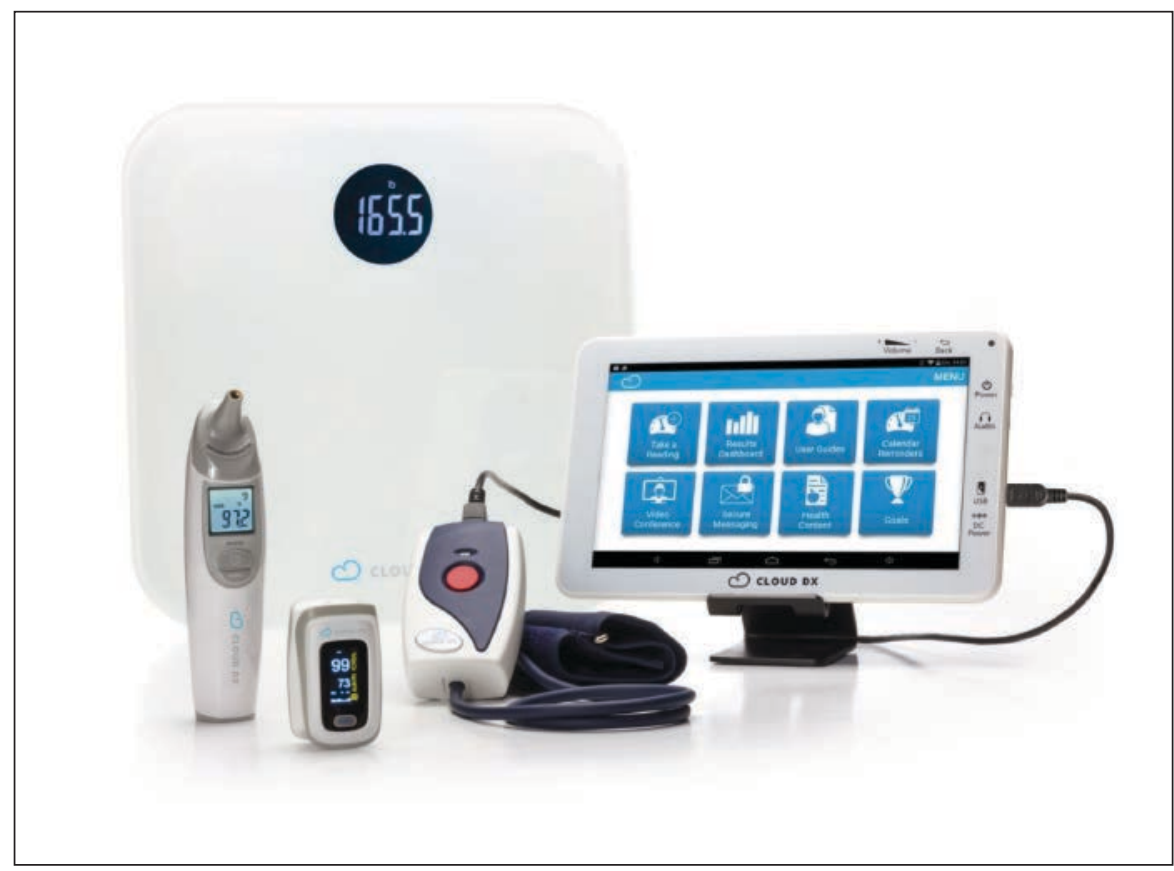

Figure 2: Cloud DX Connected Health Kit, including Bluetooth-enabled Pulsewave wrist cuff blood pressure monitor, body weight scale, wireless oximeter and temperature probe, paired with Android health tablet. Reproduced with permission from Cloud DX.

Patients in the group receiving virtual care with RAM interact daily with a "virtual nurse" (i.e., they interact with a nurse via the cellular modem-enabled tablet) on days 1-15 after randomization and every other day on days 16-30. On days without planned virtual visits, nurses organize unscheduled virtual visits if patients' biophysical measurements or recovery survey responses exceed predetermined thresholds or if the nurse identifies another reason for concern.

During virtual visits, virtual nurses discuss any patient symptoms, evaluate the patient's wound and obtain a picture of it, and reinforce principles related to recovery after surgery and the need for physical distancing. On days $1,8,15$, 22 and 30 after randomization, virtual nurses also undertake medication review and reconciliation. Virtual nurses escalate care to preassigned and available physicians (i.e., perioperative physicians or surgeons) if patients' RAM measurements exceed predetermined thresholds (Appendix 1D), patients report specific symptoms (e.g., syncope), drug errors are identified or the virtual nurses have concerns about patients' health that they cannot resolve. Physicians will add or modify treatments as indicated and, if required, have patients come to an outpatient facility for evaluation or management. Patients have access to a nurse or physician 24 hours a day, 7 days per week.

In the standard care group, patients receive management after their hospital stay as per the usual care at the hospital in which they underwent surgery. No changes to surgeons' usual care regarding postdischarge management are made for patients randomly assigned to the standard care group, as a result of participation in the trial. Patients commonly follow up with surgeons between 2 and 8 weeks after hospital discharge.

\section{Trial outcomes}

The initial primary outcome was acute hospital care; however, with 1 of the first patients randomly assigned to receive virtual care, we recognized the potential for a competing outcome issue with this outcome (Appendix 1E). We therefore changed the primary outcome to number of days alive at home during the first 30 days after randomization.

Secondary outcomes during the first 30 days after randomization include hospital readmission, emergency department visit, urgent care centre visit, acute hospital care, brief acute hospital care, all-cause hospital days, medication error detection, medication error correction and death. An additional secondary outcome is pain, assessed at 7, 15 and 30 days after randomization. Tertiary outcomes and outcome definitions are reported in appendices $1 \mathrm{~F}$ and $1 \mathrm{G}$. We hypothesize that virtual care with RAM will improve the primary, secondary and tertiary outcomes. We expect to detect more medication errors in the intervention group than in the control group and would interpret this as an improvement in care.

\section{Follow-up}

The day of randomization is day 0 of follow-up, the day after randomization is day 1 of follow-up after randomization, and so on. Because patients are followed from the day of randomization (i.e., day 0 of follow-up) until day 30 after randomization, patients have 31 days of follow-up. If study personnel are unsuccessful in contacting patients, they contact the patient's primary care physician or a close relative or friend not residing with the patient, whose contact information the patient provides at the time of enrolment. If patients (or next of kin) indicate that the patients have experienced an outcome, we contact their physicians to obtain documentation. The followup process for patients in the virtual care and standard care groups is presented in Appendix $1 \mathrm{H}$.

\section{Data management}

Study personnel and virtual nurses record data on case report forms and submit the forms through a secure computerized database (iDataFax) via the Internet. Patients are identified using a unique numeric code and all patient data are anonymized to ensure confidentiality. Data validity checks are preprogrammed in the database and are monitored by data management assistants from the project office through multi-level data validation of case report forms.

\section{Sample size}

Table 2 reports the trial power on the basis of a 2 -sided $\alpha$ of 0.05 and a sample size of 450 patients in each study group. We 
expect patients in the control group to have, on average, 29.60 days alive at home, of 31 potential days. If, on average, virtual care with RAM results in 29.81 days alive at home, we will have $89 \%$ power. An additional 0.21 days alive at home (i.e., the difference between the 2 study groups) in the virtual care with RAM group corresponds to an additional day alive and out of hospital for each 5 patients assigned to virtual care with RAM. For other possible estimates of the number of days alive at home in the control group (i.e., 29.40, 29.50, 29.60), for absolute increases of 0.21 to 0.30 days alive at home in the intervention group, we have $89 \%-99 \%$ power.

\section{Trial organization}

The PHRI is the sponsor and coordinating centre for this trial and is primarily responsible for the organization of the trial, the development of the randomization scheme, the study database, data consistency checks, data analysis and coordination of the study centres. The trial structure includes the following groups: project office, operations committee, steering committee, data monitoring committee (DMC), site principal investigators, co-investigators and adjudication committee.

\section{Patient and public involvement}

A panel of 4 patient partners reviewed the daily symptom survey for clarity and perceived ease of use. Given rules on social distancing and limitations to in-person meetings, all feedback was provided via email. We will conduct semistructured interviews with study patients and family members, nurses and physicians to understand the experience of the PVC-RAM intervention. ${ }^{8,9}$ Interview questions will focus on the recovery experience through PVC-RAM, compared with the experience of patients and family members with surgical recovery in the past or compared with the experience of nurses and physicians caring for surgical patients previously. Inductive thematic analysis will be used to code and analyze these data, with NVivo used to facilitate analysis. ${ }^{10}$ We plan to interview up to 20 nurses, 20 physicians, and 20 patients and families but will cease once data saturation is reached. ${ }^{11}$

Table 2: Power using 2-sided $\alpha$ of 0.05 , with 450 subjects per arm

\begin{tabular}{|llcc|}
\hline & \multicolumn{2}{c}{$\begin{array}{c}\text { No. of days that patients are } \\
\text { alive at home; study group }\end{array}$} & \\
\cline { 2 - 3 } Scenario & $\begin{array}{c}\text { Standard } \\
\text { care }\end{array}$ & $\begin{array}{c}\text { Virtual care } \\
\text { with RAM }\end{array}$ & $\begin{array}{c}\text { Power, } \\
\%\end{array}$ \\
\hline 1 & 29.40 & 29.61 & 89 \\
\hline 2 & 29.40 & 29.69 & 99 \\
\hline 3 & 29.50 & 29.71 & 89 \\
\hline 4 & 29.50 & 29.80 & 99 \\
\hline 5 & 29.60 & 29.81 & 89 \\
\hline 6 & 29.60 & 29.90 & 99 \\
\hline $\begin{array}{l}\text { Note: RAM = remote automated monitoring. Power calculations were based on } \\
\text { the Poisson comparison of 2 means [PASS software subroutine]. }\end{array}$ \\
\hline
\end{tabular}

\section{Trial progress}

The first patient was randomly assigned to a study group on Apr. 23, 2020, and recruitment of 905 patients was completed on July 25, 2020. The DMC (described in Appendix 1) undertook the first scheduled interim analysis and recommended continuation of the trial; however, the second scheduled interim analysis did not occur because recruitment of the last $25 \%$ of participants occurred before the first $75 \%$ of participants completed their 31-day follow-up.

\section{Statistical analysis}

Following the intention-to-treat principle, we will analyze data for patients in the study groups to which they were randomly assigned. The operations committee will create a separate statistical analysis plan that the statistical analyses will follow. The statistical analysis plan will be finalized before any investigator is unblinded to the trial results.

\section{Main analyses}

For the primary analysis, we will use Poisson regression with random intercept effects for centre to account for residual effects of centres, to estimate the 31-day effect of virtual care and RAM technology compared with standard care on the primary outcome of number of days alive at home. In this model, we will adjust for the type of surgery and prerandomization variables known to be associated with acute hospital care after discharge following surgery. For the primary outcome, we will use an exact version of the Mann-Whitney-Wilcoxon test to establish the $p$ value. We will infer statistical significance if the computed 2 -sided $p$ value is less than an $\alpha$ of 0.05 .

For the binary secondary and tertiary outcomes, we will compare the effect of virtual care and RAM technology using modified Poisson regression, ${ }^{12}$ and we will report the corresponding relative risk reductions or increases and $95 \%$ confidence intervals. For continuous outcomes, we will evaluate intervention effects using the regression approach to fitting the analysis of covariance models, so we can obtain estimates and their $95 \%$ confidence intervals for the independent variables. In these models, we will adjust for the type of surgery (i.e., cardiac v. noncardiac) and will also evaluate inclusion of prerandomization variables known to be associated with acute hospital care after discharge following surgery.

\section{Interim analyses}

Two interim analyses based on the primary outcome will occur when $50 \%$ and $75 \%$ of the patients have been followed for 30 days after randomization. The DMC will use the modified Haybittle-Peto rule of 4 standard deviations $(\alpha=$ 0.00006 , corresponding to a difference in the primary outcome of 0.32 days with $83 \%$ power) for the first planned interim analysis and 3.5 standard deviations $(\alpha=0.00047$, corresponding to a difference in the primary outcome of 0.29 days with $92 \%$ power) for the second planned interim analysis. For a finding of the intervention to be considered significant, these predefined boundaries will have to be exceeded in at least 2 consecutive analyses, 2 or more months apart. The $\alpha$ level for the final analysis will remain at the conventional 
value of 0.05 given the infrequent interim analyses, their extremely low $\alpha$ levels, and the requirement for confirmation with subsequent analyses.

At any time during the trial, if safety concerns arise the DMC chairperson will assemble a formal meeting of the full committee. The DMC will make their recommendations to the operations committee after considering all available trial data and any external data from relevant studies. If a recommendation for termination is being considered, the DMC will invite the operations committee to explore all possibilities before a decision is made. A detailed charter has been developed and governs the activities of the DMC. The DMC members have expertise in clinical trials, perioperative medicine and biostatistics.

\section{Ethics approval}

We require documentation of research ethics committee or institutional review board approvals before sites are activated to enrol patients. Investigators are informed of protocol amendments, and research ethics committees and institutional review boards are asked to approve them. Research personnel obtain informed consent for each patient before randomization. Data are stored on a central encrypted, high-security computer system and kept strictly confidential.

\section{Interpretation}

Hospitals need to facilitate management of the backlog of patients waiting for elective surgery, ensure hospital capacity for patients with COVID-19 and minimize the spread of COVID-19. Hospitals have a continuing obligation to treat patients with semiurgent, urgent or emergent conditions who do not have COVID-19. After discharge following nonelective surgery, these patients are at high risk (i.e., $15 \%-$ $25 \%$ ) of needing subsequent acute hospital care and of dying. ${ }^{1-3}$ The PVC-RAM intervention is a proposed innovative strategy that can potentially meet these challenges.

Planned knowledge translation activities are as follows. Elsevier has developed an integrated, online, multimedia resource centre as a platform for large-scale knowledge dissemination; $;^{13}$ we will include on this site multimedia slide and audio programs, patient testimonial interviews and fact sheets related to the results of PVC-RAM. We will write a policy brief supporting evidence-informed transformations at all levels of the health system, which includes a synthesis of research and systematic reviews. We will also give presentations at national and international conferences and write publications for peer-reviewed journals, and we hope to garner national coverage of the study through a high-impact media release of the trial results.

\section{Limitations}

A potential challenge for trials employing virtual care with RAM is patient noncompliance with intervention protocols including provision of daily vital signs measurements. ${ }^{14}$ However, it is anticipated that immediate, real-time access to clinicians and support will encourage patients to continue to participate in the intervention.

\section{Conclusion}

A strong rationale and promising data suggest that virtual care with RAM technology can increase number of days alive at home, among adults discharged following nonelective surgery. The PVC-RAM trial will provide important information on how to manage surgical patients after discharge in the setting of a pandemic and offer insights for the management of patients who undergo nonelective surgery in a nonpandemic setting.

\section{References}

1. All-cause readmission to acute care and return to the emergency department. Ottawa: Canadian Institute for Health Information; 2012.

2. Glance LG, Kellermann AL, Osler TM, et al. Hospital readmission after noncardiac surgery: the role of major complications. 7AMA Surg 2014;149:439-45.

3. Vascular Events in Noncardiac Surgery Patients Cohort Evaluation (VISION) Study Investigators. Association between complications and death within 30 days after noncardiac surgery. CMAJ 2019;191:E830-7.

4. Yang H, Dervin G, Madden S, et al. Postoperative home monitoring after joint replacement: retrospective outcome study comparing cases with matched historical controls. 7MIR Perioper Med 2018;Jul-Dec;1:e10169.

5. Devereaux PJ, Yang H, Yusuf S, et al. Effects of extended-release metoprolol succinate in patients undergoing non-cardiac surgery (POISE trial): a randomised controlled trial. Lancet 2008;371:1839-47.

6. Devereaux PJ, Mrkobrada M, Sessler DI, et al. Aspirin in patients undergoing noncardiac surgery. N Engl 7 Med 2014;370:1494-503.

7. Lefler LL, Rhoads SJ, Harris M, et al. Evaluating the use of mobile health technology in older adults with heart failure: mixed-methods study. $7 M I R$ Aging 2018;1:e12178.

8. Bradshaw C, Atkinson S, Doody O. Employing a qualitative description approach in health care research. Glob Qual Nurs Res 2017;4:2333393617742282.

9. Sandelowski M. Whatever happened to qualitative description? Res Nurs Health 2000;23:334-40.

10. Braun V, Clarke V. Using thematic analysis in psychology. Qual Res Psychol 2006;3:77-101.

11. Marshall B, Cardon P, Poddar A, et al. Does sample size matter in qualitative research? A review of qualitative interviews in IS research. 7 Comput Inf Syst 2013;54:11-22.

12. Zou G. A modified Poisson regression approach to prospective studies with binary data. Am 7 Epidemiol 2004;159:702-6.

13. Devereaux PJ, McGillion M. Reducing global perioperative risk. New York: Elsevier; 2017. Available: http://perioperative-risk.amjmed.com (accessed 2020 Dec. 21).

14. Bertoncello C, Colucci M, Baldovin T, et al. How does it work? Factors involved in telemedicine home-interventions effectiveness: a review of reviews. PLoS One 2018;13:e0207332.

Competing interests: CloudDX undertook training sessions for study nurses, perioperative physicians and surgeons regarding how to use their technology. David Conen has received personal fees from Servier Canada, outside of the current work. Emil Schemitsch has received personal fees from Stryker, Smith \& Nephew, ITS Implants, Acumed, Swemac and DePuy Synthes, outside the present work. Emilie Belley-Cote has received grants from Bayer and Roche, outside the present work. Richard Whitlock has received grants from Bayer, Roche and Boehringer Ingelheim, an honorarium from Boehringer Ingeheim and consulting fees from AtriCure and PhaseBio, outside the present work. P.J. Devereaux has received a grant from Roche Diagnostics for the present work and grants from Abbott Diagnostics, Boehringer Ingeheim, Roche Diagnostics and Siemens, outside the present work, as well as patient monitors from Philips Healthcare and troponin assays from Siemens, outside the present work.

Affiliations: School of Nursing (McGillion, Levesque, Lounsbury, Bird, Ouellette), McMaster University, Hamilton, Ont.; Population Health Research Institute (McGillion, Whitlock, Devereaux, Belley-Cote, Borges, Vincent, Lamy, Bangdiwala, Le Manach, Harvey, Conen), Hamilton, Ont.; Department of Anesthesiology and Perioperative Medicine (Parlow, Arellano), Queen's University, Kingston, Ont.; Department of Anesthesiology and Perioperative Medicine (Parlow, Shelley, DuMerton, Arellano), Kingston Health Sciences Centre, Kingston, Ont.; Department of Medicine (Belley-Cote, Yousef, Borges, Marcucci, Chaudhry, Haider, Guyatt, Patel, Conen, Tandon), McMaster University, Hamilton, Ont.; Department 
of Health Research Methods, Evidence, and Impact (Whitlock, Devereaux, Marcucci, Bangdiwala, Guyatt), McMaster University, Hamilton, Ont.; Departments of Critical Care and Anesthesiology (Jacka), University of Alberta, Edmonton, Alta.; Department of Surgery (Adili, Harlock, Simunovic, Nenshi), McMaster University, Hamilton, Ont.; St. Joseph's Healthcare Hamilton (Adili, LeBlanc, Nenshi, Tandon), Hamilton, Ont.; Department of Anesthesiology and Pain Medicine (Lalu, McCartney, Hamilton), University of Ottawa and The Ottawa Hospital, Ottawa, Ont.; Ottawa Hospital Research Institute (Moloo, Lalu, Halman, Forster, Hamilton), Ottawa, Ont.; Department of Anesthesia and Perioperative Medicine (Sehmbi, Nagappa, Yang), Schulich School of Medicine, University of Western Ontario, London, Ont.; Department of Anesthesia and Perioperative Medicine (Sehmbi, Nagappa, Yang), London Health Sciences Centre, London, Ont.; Hamilton Health Sciences (Meyer, Paulin, Pettit, Lamy, Lounsbury, Scott, Patel, Ouellette, O'Leary), Hamilton, Ont.; Department of Anesthesia (Shanthanna, Paul, Le Manach, O'Leary), McMaster University, Hamilton, Ont.; Department of Medicine (Roshanov, Mrkobrada), Schulich School of Medicine, University of Western Ontario, London, Ont.; Department of Internal Medicine (Ofori), University of Port Harcourt, Port Harcourt, Nigeria, West Africa; Department of Medicine (Graham, Taylor), University of Alberta, Edmonton, Alta.; Department of Medicine (Halman, Forster), University of Ottawa and The Ottawa Hospital, Ottawa, Ont.; Lawrence S. Bloomberg Faculty of Nursing (Peter), University of Toronto, Toronto, Ont.; Department of Medicine (Marosi), Queen's University, Kingston, Ont.; Alberta Health Services (Deuchar), Alberta, Canada; Department of Surgery (Parry, Schlachta, Schemitsch), University of Western Ontario and London Health Sciences Centre, London, Ont.; Department of Surgery (Williams), University of Alberta, Edmonton, Alta.; Department of Oncology (Meyer), McMaster University, Hamilton, Ont.; Department of Surgery (Pichora), Queen's University and Kingston Health Sciences Centre, Kingston, Ont.; Department of Surgery (Moloo), University of Ottawa and The Ottawa Hospital, Ottawa, Ont.; The Ottawa Hospital (Waggott), Ottawa, Ont.

Contributors: All authors planned the conceptualization and design of the study and the protocol. All authors contributed to critical revisions of the manuscript and approved the final version to be published. All authors agreed to be accountable for all aspects of this work.

PVC-RAM Investigators: Participating centres and investigators: Kingston Health Sciences: Darrin Payne, Rachael DaCunha, Sunil Patel, Michael Yacob, Siddhartha Srivastava, Lisa Nguyen, Curtis Nickel, Tyler Hands, Elorm Vowotor, Emile Peponoulas, Angela Webster, Tammy Doyle; Hamilton Health Sciences, Hamilton General Hospital: Kajenny Srivaratharajah, Dave Szalay, Deborah Bedini, Victor Chu, Jason Busse, Sandra Carroll, Jeremy Petch, Duane Bender, Dina Brooks, Krysten Gregus, Patricia Power, Dale Williams; Hamilton Health Sciences, Juravinski Hospital and Cancer Centre: Amitabha Chakroborty, Samir Raza, Amna Ahmed, Kelly Lawrence, Derek Hunt, David Cowan, Jehonathan Pinthus, David Wilson, Clare Reade, Leslie Gauthier, Stephen Kelly, Kirsten Krull, Kim Alvarado, Susan Reid, Mohit Bhandari; University of Alberta Hospital: Derek Dillane, James Greene, David Bigam, Ryan Snelgrove, Brian Buchanan, Oleksa Rewa, Ronald Brisebois, Nadr Jomha, Bruce Ritchie, Sherry Reid, Adrian Fairey, Greg Hrynchyshyn; St. Joseph's Healthcare Hamilton: Bobby Shayegan, Christian Finley, Wendy Lim, Maria Tiboni, David Choi, Anne-Marie MacDonald, Deanna Burnette, Tom Stewart, Melissa Farrell, Carolyn Goss, Faraaz Quiraishi; The Ottawa Hospital: Daniel McIsaac, Sarah Tierney, Shawn Hicks, Kathryn Wheeler, Josh Robert, Colleen McFaul, Greg Krolczyk, Purnima Rao, Stephane Moffett, Dan Dubois, Catherine Code, Heather Clark, Melissa Rousseau, Catherine Gray, Dominique Yelle, Youssef Tawil, Babak Rashidi, Weiwei Beckerleg, Shipa Gupta, Sudhir Sundaresan, Suzanne Madore, Andrew Seely, Reece Bearnes, Dean Fergusson, Susan Madden, Jad Abou Khalil, John Sinclair, Moein Momtazi, Rodney Breau, Humberto Vigil, James Chan; Freddy Nguyen, London Health Sciences (University and Victoria Hospitals): George Nicolaou, Yamini Subramani, Ashraf Fayad, Amit Garg, Cathy Vandersluis, Glen Kearns, Cheryl Churcher, Carla Cormack, Brenda Maxwell, Johana Halabi, James Calvin, Douglas Naudie, Melfort Boulton, Stephanie Handsor, Heather Whittle, Charlotte Kenning. Pop- ulation Health Research Institute Coordinating Centre: Lori Blake, Sanela Dragic-Taylor, Arielle Fernandez, Peggy Gao, Valerie Harvey, Peter Koh, Louise Mastrangelo, John Liu, Yan Yun Liu, Rajibul Mian, Wesley Tong, Jessica Vincent, Heidi Wilton. Event Adjudication Committee: Flavia K. Borges (Chair), Sandra Ofori, Michael Wang, James Khan, Rahima Nenshi, Maura Marcucci.

Funding: This trial is supported by the following grants: Roche COVID19 Open Innovation Challenge Application grant; McMaster COVID-19 Research Fund grant; Research Institute of St. Joseph's Healthcare Hamilton grant; COVID-19 Innovation Fund grant from the Ottawa Hospital Academic Medical Association; and Queen's University Department of Anaesthesiology award and Department of Medicine research award to help fund the trial at Kingston Health Sciences. This trial received inkind support to cover the salaries of the virtual nurses from Hamilton Health Sciences, Kingston Health Sciences, London Health Sciences, St. Joseph's Healthcare Hamilton, The Ottawa Hospital, and the University of Alberta Hospital. Michael McGillion holds the Heart and Stroke Foundation - Michael G. DeGroote Endowed Chair in Cardiovascular Nursing. Carley Ouellette, Kelsea Levesque and Sandra Ofori received internship funding from Mitacs Accelerate to support work on the PVC-RAM trial. Flavia Borges and Maura Marcucci hold McMaster University Department of Medicine career research awards. David Conen holds a McMaster University Department of Medicine mid-career research award. P.J. Devereaux holds the McMaster University Hamilton Health Sciences Chair in Perioperative Care and a Tier 1 Canada Research Chair in Perioperative Medicine.

Content licence: This is an Open Access article distributed in accordance with the terms of the Creative Commons Attribution (CC BY-NC-ND 4.0) licence, which permits use, distribution and reproduction in any medium, provided that the original publication is properly cited, the use is noncommercial (i.e., research or educational use), and no modifications or adaptations are made. See: https://creativecommons.org/licenses/ by-nc-nd/4.0/

Data sharing: The Population Health Research Institute (PHRI) is the sponsor of this trial. The PHRI believes the dissemination of clinical research results is vital and sharing of data is important. PHRI prioritizes access to data analyses to researchers who have worked on the trial for a substantial duration, have played substantial roles and have participated in raising the funds to conduct the trial. PHRI balances the length of the research study and the intellectual and financial investments that made it possible with the need to allow wider access to the data collected. Data will be disclosed only upon request and approval of the proposed use of the data by a review committee. Data are available to journals considering publication of trial results. Data requests from other non-PVC-RAM investigators will not be considered until 5 years after the close out of the trial. Regarding the ICES data, while data sharing agreements prohibit ICES from making the data set publicly available, access can be granted to those who meet prespecified criteria for confidential access, available at www.ices.on.ca/DAS.

Dedication: We dedicate this paper to the memory of Dr. Yannick Le Manach, who was a colleague and PVC-RAM investigator. Yannick was a brilliant biostatistician, clinical epidemiologist and anesthesiologist. Unfortunately, depression cut his life short. His tragic loss highlights the need for more trials to inform how to better prevent and manage depression and suicide.

Disclaimer: Additional data from ICES will be used in the 6-month analyses. ICES is funded by an annual grant from the Ontario Ministry of Health and Long-Term Care (MOHLTC). The members of the writing committee are solely responsible for the trial design, conduct, management, analyses, data interpretation, writing of this paper, and decision to submit this paper for publication. No endorsement by ICES or the Ontario MOHLTC is intended or should be inferred.

Supplemental information: For reviewer comments and the original submission of this manuscript, please see www.cmajopen.ca/content/9/1/ E142/suppl/DC1.

Editor's note: This study protocol was submitted before participant recruitment was completed. 